\title{
A path analysis of multiple neurotoxic chemicals and cognitive functioning in older US adults (NHANES 1999-2002)
}

Jennifer Przybyla* ${ }^{*}$, E. Andres Houseman, Ellen Smit and Molly L. Kile

\begin{abstract}
Background: Polychlorinated biphenyls (PCBs) and metals (lead and cadmium) are neurotoxic and affect neurobehavioral performance. Yet little is known about the association between exposure to multiple neurotoxic compounds and cognitive functioning in older adults.

Methods: Using data from two consecutive cycles of the National Health and Nutrition and Examination Survey (1999-2002), path analysis was used to simultaneously evaluate the association between whole blood concentrations of 14 neurotoxic compounds and cognitive functioning measured by the Digit Symbol Coding Test of the Weschler Adult Intelligence Scale, $3^{\text {rd }}$ Edition in participants 60-84 years of age $(N=498)$. Effect modification was assessed for age (above/below the mean) and sex.

Results: The final path model fit 5 compounds (i.e. PCB 74, PCB 118, PCB 146, PCB 153, and lead). After controlling for co-exposures and confounders, PCB 146 ( $\beta=-0.16,95 \% \mathrm{Cl}:-0.29,-0.02, p=0.02)$ and lead $(\beta=-0.10$, $95 \% \mathrm{Cl}:-0.20,-0.006, p=0.04)$ were negatively associated with DSC scores in 60-84 year olds. Whereas, PCB 153 was positively associated with DSC scores ( $\beta=0.20,95 \% \mathrm{Cl}: 0.05,0.35 ; p=0.01$ ).

Conclusions: This cross-sectional analysis which controlled for collinear exposure to several neurotoxic compounds demonstrated an association between non-dioxin like polychlorinated biphenyl exposure, specifically PCB 146, and lower cognitive functioning, in older adults. Lead exposure was also weakly associated with lower cognitive functioning. Additional studies are needed to determine the causality of the observed associations.
\end{abstract}

Keywords: Polychlorinated biphenyls, PCBs, Lead, Cadmium, Cognitive decline, Path analysis, Digit symbol coding test

\section{Background}

Exposure to certain industrial chemicals such as lead, cadmium, and polychlorinated biphenyls (PCBs) can affect the central nervous system (CNS) and result in alterations in neurobehavioral performance [1-3]. Once absorbed in the body lead, cadmium, and PCBs can influence brain functioning by affecting neural cells, inducing oxidative stress, and lowering dopamine concentrations [4-6]. Agerelated decreases in cognitive functioning is typical as life progresses, mild cognitive impairment (MCI) is a greater than average age-related change in cognitive functioning [7]. Because the US population is rapidly aging and agerelated cognitive impairment are predicted to have a high

\footnotetext{
* Correspondence: jennifer.przybyla@oregonstate.edu

School of Biological and Population Health, College of Public Health and Human Sciences, 101 Milam Hall, Corvallis, OR 97330, USA
}

societal and economic impact [8-10], identifying preventable environmental exposures is important to ensure a healthy aging population.

Considerable evidence shows that the developing brain is vulnerable to environmental pollutants [11-15]. There is also evidence that the brain is vulnerable to environmental toxics during the later stages of life due to behavioral, metabolic and physiological changes that occur with aging [16]. These studies are often restricted to examining the effect of single environmental exposures on neurocognitive outcomes in older adults. For instance, the Normative Aging Veterans Affair (VA) cohort demonstrated that blood lead levels were a significant negative predictor of performance on speed memory, spatial copying and vocabulary $(n=141)$ from a battery of 8 cognitive tests [17]. Lead was associated with higher 
odds of having a Mini Mental State Exam (MMSE) score $(n=1,031)$ less than 24 , which is an indication of increased risk of dementia in elderly men [18]. Cadmium also had a negative association with cognitive functioning using Symbol Digit Substitution Test (SDST) in US adults 20-59 $(n=5,572)$ [19]. Whereas another study with Chinese subjects $>65$ years of age $(n=1,016)$ observed a negative relationship between cadmium exposure and cognitive functioning using a composite score of the Community Screening Instrument for Dementia (CSID), the Consortium to Establish a Registry for Alzheimer's Disease (CERAD), Word List Learning Test, the CERAD Word List Recall Test, the Indiana University (IU) Story recall, Animal Fluency test and the IU Token test [20]. Total serum polychlorinated biphenyls (PCBs) have also been associated with decreased measures of memory and learning as measured by the California Verbal Learning Test trial and increase depression as measured by the Beck Depression Inventory in participants $(n=253)$ aged 55-74 years of age living in the vicinity of form capacitor plants in Hudson Falls and Fort Edward, NY [21].

Yet very little is known about how exposure to multiple environmental toxics affect cognition in adults despite the fact that humans are often exposed to mixtures from several different chemical classes. Attempts have been made to examine the effect of PCB mixtures on cognitive functioning, either summing PCB concentrations or using toxic equivalency factors (TEFs). TEFs report the toxicity of a single $\mathrm{PCB}$ congener in relation to the most toxic dioxin, 2,3,7,8-TCDD [22]. TEFs are determined using relative effect potencies (REP) established using World Health Organization (WHO) criteria of a compound's binding capacity and ability to elicit toxic responses from the AhR, persistence in the environment, and accumulation in food chain [23]. Summing concentrations of different PCBs congeners assumes an additive nature of the chemicals specific to the endpoint, which in the case of PCBs and cognitive functioning may or may not be true. Additionally using the TEFs to summarize the potency of PCBs mixtures must be done with caution considering PCBs' TEFs are both species and response dependent [24].

Therefore, we employed path analysis to examine the association between multiple chemical exposures from different chemical classes on cognitive functioning in older adults. Path analysis is a technique well-suited to modeling multiple environmental exposures because of the ability to determine the magnitude and significance of the relationship between several exposures and an outcome simultaneously while adjusting for multiple comparisons $[25,26]$. We hypothesized that exposure to multiple environmental chemicals would be negatively associated with cognitive functioning in older U.S. adults as measured by the Digit Symbol Coding (DSC) test from the Wechsler Adult Intelligence Scale, $3^{\text {rd }}$ edition (WAIS-III).

\section{Methods}

\section{Study design and population}

Data from the National Health and Nutrition Examination Survey (NHANES) continuous cycles 1999-2000 and 2001-2002 were merged for this analysis. NHANES is funded and conducted by the National Center for Health Statistics (NCHS), which is part of the Center for Disease Control (CDC). NHANES is the main tool used to gather data to guide federal health programs and initiatives. NHANES collects data by utilizing physical examinations, specimen collection and surveys to collect data on nutrition and health measurements from a US noninstitutionalized representative sample population [27].

This study focused on older adults aged $\geq 60$ years. For confidentiality reasons, NHANES top-codes age at 85 years of age. Therefore, to eliminate outliers due to extreme age and to be consistent with previous studies [28] this sample consists of women and men aged 60-84 years of age who had their blood samples analyzed for lead, cadmium and PCBs and completed the WAIS-III DSC module $(n=870)$. Since having a stroke is a major reason for cognitive dysfunction [29], participants were excluded $(n=57)$ from the analysis if they answered yes to the question "Has a doctor or other health professional ever told you that you had a stroke?"

Of the 813 individuals aged 60-84 year in the subsample that had their blood serum analyzed for PCBs and reported not having a stroke, 715 individuals also had information on DSC scores. However, 102 individuals were missing information on sociodemographic variables with 95,6 , and 1 individuals missing data on poverty income ratio (PIR), smoking status, and education, respectively. Among participants with measured PCB, 115 individuals were excluded due to potential contamination or inadequate biospecimen sample size leaving 498 individuals with complete data [30].

\section{Exposure}

PCBs were measured in blood serum using high-resolution gas chromatography/isotope-dilution. Cadmium and lead were measured in blood samples using inductively coupled plasma mass spectrometry (ICP-MS) [31]. The methods for detecting the environmental chemicals did not change from the 1999-2000 to the 2001-2002 cycles. All analytes were detected at a frequency of $75 \%$ or above. For chemical values detected below the limit of detection (LOD), NCHS imputes values equal to the limit of detection divide by the square root of $2(\mathrm{LOD} / \sqrt{ } 2)$ into the dataset.

\section{Cognitive functioning}

Cognitive functioning was assessed using the DSC Module of the WAIS-III. Participants were given a key with symbols corresponding to letters. It was ensured the participants had an adequate writing area, glasses (if 
needed) and could complete the test without distraction before they were allowed to begin the test. Practice sheets were given to ensure the concept of the test was understood before proceeding with the test. Participants were then given the test sheet which showed numbers and asked to draw as many of the corresponding symbols as they could in $120 \mathrm{~s}$. The number of symbols correctly drawn were then summed with a maximum score of 133. Cognitive scores were not provided for participants who refused to take the test, could not complete the test due to distraction, had cognitive or physical limitations, or did not complete the test in the given time limit. Examiners were given extensive instruction on how to score the symbol drawing and $10 \%$ of the test were scored twice as quality assurance, all scores were consistent [32]. Cognitive testing procedure did not change from the 1999-2000 to the 2001-2002 cycles.

\section{Covariates}

We explored a variety of sociodemographic covariates based on prior literature showing that they were related to cognitive functioning and/or environmental chemical exposure including: race/ethnicity (Mexican American [MA], Other Hispanic [OH], Non-Hispanic White [NHW], NonHispanic Black [NHB]), age (continuous), education level (less than $9^{\text {th }}$ grade, $9-11^{\text {th }}$ grade, high school diploma or GED, some college, college graduate or above), PIR $(\leq 0.99$ and $\geq 1.00$ ) and sex (male/female) [28, 33-35]. Since cigarette smoke is a source of cadmium and lead [36] and has been associated with decreased cognitive functioning [37], smoking status was explored as a covariate. Participants with blood cotinine levels $\leq 10 \mathrm{ng} / \mathrm{L}$ were categorized as non-smoking and participants with blood cotinine levels of $>10 \mathrm{ng} / \mathrm{L}$ were categorized as smokers [38].

\section{Statistical analysis}

All chemical exposures were natural log transformed to address skewness. Descriptive statistics were calculated using a 4 year sampling weight to account for using two consecutive cycles of NHANES using Stata for Windows (version 14, StataCorp LP, http://www.stata.com/). Survey variables including stratification, clustering (PSU) and sample weights corresponding to the 4 year weights of the subsample who had their serum analyzed for PCBs were used to account for the complex sampling design of the NHANES [39].

Path analysis was conducted using MPlus (version 7.4, Muthén and Muthén; http://www.statmodel.com/) to determine the standardized path coefficients, although Stata was used to construct the path diagram. Survey variables and sampling weights were included in the path analysis. The path coefficients in a standardized model can be used to compare the relative influence among variables that have different measurements scales and are similar to beta coefficients in regression. In path analysis the term "effect" refers to statistical effect not causal effect. Complete case analysis was used.

Initially 14 chemicals from the chemical groups of metals (lead and cadmium), non-dioxin like PCBs (PCB 74, РCB 99, РCB 138, РCB 146, РCB 153, РCB 170, PCB 180, PCB 187), and dioxin-like PCBs (PCB 118, PCB 126, PCB 156, PCB 169) were included in the a priori path analysis models (Fig. 1). A more parsimonious model was then fit by removing environmental chemicals that were not significantly ( $p$-value $\geq 0.05$ ) associated with cognitive functioning. Models were adjusted for PIR, education, race, age, sex and smoking status. Multiple group analysis along with the Wald Chi-Square test was used to assess effect modification by age (above mean/below mean) and sex because previous studies have reported differences in associations

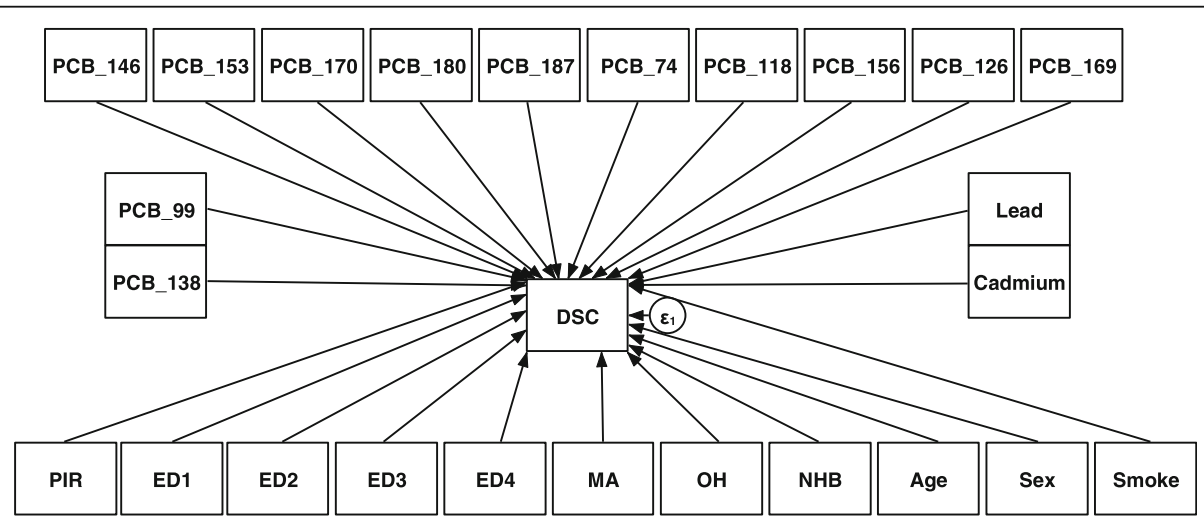

Fig. 1 A priori model of 14 neurotoxic compounds and DSC scores and confounding factors. PIR is poverty index ratio, Ed1 is less than 9th grade, Ed2 is High School Grad/GED, Ed3 is Some College or AA degree, Ed4 is College Graduate or above, MA is Mexican American, OH is Other Hispanic and NHB is Non-Hispanic Black 
between PCBs and neuropsychological functioning by age and sex [40].

We conducted a sensitivity analysis by re-analyzing the final models using non-lipid adjusted PCBs instead of lipid-adjusted PCBs. This is because the serum samples collected in this study were non-fasting which can influence the concentration of lipophilic compounds [41]. A second sensitivity analysis was conducted to address missingness using Markov Chain Monte Carlo (MCMC) simulations to create 20 complete data sets with imputed values for the missing data.

\section{Results}

The descriptive characteristics of the population are shown in Table 1. We compared the characteristics of the subpopulation included in this analysis with those who were excluded due to missing data. We observed that the individuals included in this analysis were more likely to be Mexican American, Other Hispanic or NonHispanic White. Otherwise, the characteristics of the subsample included in this analysis were similar to those with missing data.th $=$ tlb=

The mean concentrations for each of the biomarkers are shown in Table 2. The mean concentration of lead and cadmium was $2.17 \mu \mathrm{g} / \mathrm{dL}$ (95\% Confidence Interval (95\% CI): 2.07, $2.28 \mu \mathrm{g} / \mathrm{dL}$ ) and $0.49 \mu \mathrm{g} / \mathrm{dL}(95 \% \mathrm{CI}$ : $0.46,0.52 \mu \mathrm{g} / \mathrm{dL}$ ), respectively. For the lipid adjusted and non-lipid adjusted non-dioxin like PCBs, the highest concentration was for PCB 153 with a geometric mean of $66.99 \mathrm{ng} / \mathrm{g}$ (95\% CI: 63.66, $70.50 \mathrm{ng} / \mathrm{g}$ ) and $0.44 \mathrm{ng} / \mathrm{g}$ (95\% CI: $0.42,0.47 \mathrm{ng} / \mathrm{g}$ ), respectively. For the dioxin like PCBs, PCB 118 had the highest lipid adjusted and nonlipid adjusted geometric mean of $20.16 \mathrm{ng} / \mathrm{g}$ (95\% CI: $18.43,22.05 \mathrm{ng} / \mathrm{g})$ and $0.13 \mathrm{ng} / \mathrm{g}$ (95\% CI: $0.12,0.14 \mathrm{ng} /$ g), respectively.

Our a priori model included all 14 environmental chemicals in the model. Not all environmental chemicals were significantly associated with cognitive functioning and subsequently dropped from the final path model, which included 5 different chemicals. For all participants (aged 60-84), PCB 146 had the strongest negative association with cognitive functioning scores with a path coefficient of -0.16 (95\% CI: $-0.29,-0.02)$ after adjusting for age, sex and smoking status. This effect size can be interpreted as an increase in 1 standard deviation (SD) in the exposure of PCB 146 is associated with a decrease in DSC score of 0.16 points after controlling for other chemical exposures, race, smoking, age and education. Blood lead levels were also associated with a slightly lower DSC score although the strength of this association was weaker $(\beta=-0.10,95 \%$ CI: $-0.18,-0.001, p=0.04)$. We also observed that PCB 153 had a positive association with cognitive functioning scores with a path coefficient of $0.20(95 \%$ CI: 0.05, 0.35) (Fig. 2, Table 3).
Table 1 Descriptive characteristics for adults 60-84 years of age who had their blood samples analyzed for PCBs, lead and cadmium and did not have a stroke (NHANES 1999-2002)

\begin{tabular}{|c|c|c|c|}
\hline \multirow[t]{2}{*}{ Variable } & $\begin{array}{l}\text { Males and females } \\
\text { aged } 60-84 \\
\text { who had } \\
\text { completed data }\end{array}$ & $\begin{array}{l}\text { Males and females } \\
\text { aged } 60-84 \\
\text { who did not have } \\
\text { complete data }\end{array}$ & \multirow[t]{2}{*}{$x^{2}$ (pvalue) } \\
\hline & $N\left(\%^{a}\right)$ & $N\left(\%^{a}\right)$ & \\
\hline Age (years) & & & $0.12(0.73)$ \\
\hline $60-69$ & $250(50.24)$ & $162(54.00)$ & \\
\hline $70-84$ & $248(49.76)$ & $153(46.00)$ & \\
\hline Race & & & $8.77(0.03)$ \\
\hline$M A^{b}$ & $106(2.98)$ & $76(.53)$ & \\
\hline $\mathrm{OH}^{c}$ & $35(8.24)$ & $18(6.78)$ & \\
\hline$N H W^{d}$ & $293(82.02)$ & 160 (78.97) & \\
\hline $\mathrm{NHB}^{\mathrm{e}}$ & $64(6.75)$ & $61(10.71)$ & \\
\hline PIR (range) & & & $2.23(0.14)$ \\
\hline $1^{\text {st }}$ tertile & $166(28.63)$ & 79 (19.93) & \\
\hline $2^{\text {nd }}$ tertile & 167 (32.02) & $70(22.42)$ & \\
\hline $3^{\text {rd }}$ tertile & 165 (39.35) & $57(22.58)$ & \\
\hline Missing & NA & 109 (35.07) & \\
\hline Sex & & & $0.12(0.73)$ \\
\hline Male & $231(43.45)$ & $150(43.00)$ & \\
\hline Female & 267 (56.55) & $165(57.01)$ & \\
\hline Smoking Status & & & $0.08(0.77)$ \\
\hline $\begin{array}{l}\text { Smoker } \\
\text { (cotinine > } 10 \text { ng/L) }\end{array}$ & $413(81.01)$ & $253(80.28)$ & \\
\hline $\begin{array}{l}\text { Non-smoker } \\
\text { (cotinine } \leq \\
10 \mathrm{ng} / \mathrm{L})\end{array}$ & 85 (18.99) & $55(17.87)$ & \\
\hline Missing & NA & $7(1.85)$ & \\
\hline Education & & & $6.81(0.15)$ \\
\hline $\begin{array}{l}\text { Less than } 9^{\text {th }} \\
\text { Grade }\end{array}$ & 120 (13.09) & $91(12.90)$ & \\
\hline $9-11^{\text {th }}$ Grade & $80(16.01)$ & $58(18.70)$ & \\
\hline $\begin{array}{l}\text { High School } \\
\text { Grad/GED }\end{array}$ & $121(30.25)$ & $63(25.83)$ & \\
\hline $\begin{array}{l}\text { Some College or } \\
\text { AA degree }\end{array}$ & 96 (21.39) & $65(25.41)$ & \\
\hline $\begin{array}{l}\text { College Graduate } \\
\text { or above }\end{array}$ & 81 (19.26) & $37(16.93)$ & \\
\hline Missing & NA & $1(0.22)$ & \\
\hline DSC Scores & & & $7.27(0.06)$ \\
\hline $1^{\text {st }}$ Quartile & $131(15.88)$ & $56(10.22)$ & \\
\hline $2^{\text {nd }}$ Quartile & $128(26.20)$ & $43(14.88)$ & \\
\hline $3^{\text {rd }}$ Quartile & $129(27.87)$ & $51(19.12)$ & \\
\hline $4^{\text {th }}$ Quartile & 110 (30.06) & 67 (30.69) & \\
\hline Missing & NA & $98(25.08)$ & \\
\hline
\end{tabular}

${ }^{a}$ Weighted proportion

${ }^{\mathrm{b}} M A$ Mexican American

${ }^{\mathrm{O}} \mathrm{OH}$ Other Hispanic

${ }^{\mathrm{d} N H W}$ Non-Hispanic White

${ }^{\mathrm{e}} \mathrm{NHB}$ Non-Hispanic Black 
Table 2 Description of the concentration of EDCs including geometric mean, 95\% confidence interval (95\% Cl), percent above LOD, percent who were included in this analysis (NHANES 1999-2002)

\begin{tabular}{|c|c|c|c|c|c|c|c|}
\hline Chemical & $\operatorname{LOD}^{\mathrm{a}}\left(\mathrm{LA}^{\mathrm{b}} / \mathrm{NLA} \mathrm{A}^{\mathrm{C}}\right)$ & Above LOD (\%) & Missing (\%) & Geometric Mean $(95 \%$ Cl) & Range & Geometric Mean $(95 \%$ Cl) & Range \\
\hline \multicolumn{8}{|l|}{ Metals $\mu \mathrm{g} / \mathrm{dL}$} \\
\hline Lead ( $\mu \mathrm{g} / \mathrm{dL})$ & $0.3 / \mathrm{NA}$ & 100 & 0 & $2.17(2.07,2.27)$ & $0.4-16.4$ & NA & NA \\
\hline Cadmium ( $\mu \mathrm{g} / \mathrm{L})$ & $0.3 / \mathrm{NA}$ & 86 & 0 & $0.49(0.46,0.52)$ & $0.2-4.7$ & NA & NA \\
\hline Non-Dioxin Like (ng/g) & & & & Lipid-adjusted & & Non-lipid-adjusted & \\
\hline PCB 74 & $3.95 / 0.03$ & 92 & 0.70 & $17.78(16.23,19.49)$ & $2.8-144$ & $0.12(0.11,0.13)$ & $0.02-0.89$ \\
\hline PCB 99 & $2.96 / 0.03$ & 80 & 2.10 & $10.65(9.88,11.48)$ & $2.1-132$ & $0.07(0.07,0.08)$ & $0.02-0.80$ \\
\hline PCB 138 & $2.96 / 0.03$ & 92 & 0.56 & $44.28(41.40,47.37)$ & $2.1-310$ & $0.29(0.27,0.31)$ & $0.04-4.01$ \\
\hline PCB 146 & $2.26 / 0.03$ & 74 & 1.26 & $7.86(7.39,8.35)$ & $1.6-68.6$ & $0.05(0.05,0.05)$ & $0.02-0.69$ \\
\hline PCB 153 & $2.96 / 0.03$ & 95 & 0.29 & $66.99(63.66,70.50)$ & $2.1-433$ & $0.44(0.42,0.47)$ & $0.02-5.26$ \\
\hline PCB 170 & $2.96 / 0.03$ & 91 & 4.76 & $20.59(19.76,21.46)$ & $2.1-129$ & $0.14(0.13,0.14)$ & $0.02-1.74$ \\
\hline PCB 180 & $6.20 / 0.03$ & 97 & 0.70 & $51.79(49.13,54.60)$ & $4.4-397$ & $0.34(0.32,0.36)$ & $0.02-3.65$ \\
\hline PCB 187 & $2.96 / 0.03$ & 94 & 0.14 & $14.93(14.25,15.64)$ & $2.1-178$ & $0.10(0.09,0.10)$ & $0.02-1.16$ \\
\hline Dioxin Like (ng/g) & & & & Lipid-adjusted & & Non-lipid-adjusted & \\
\hline PCB 118 & $2.96 / 0.03$ & 93 & 0.42 & $20.16(18.43,22.05)$ & $2.1-361$ & $0.13(0.12,0.14)$ & $0.02-1.95$ \\
\hline PCB126 ${ }^{d}$ & $2.54 / 21.73$ & 75 & 11.19 & $33.55(29.98,37.55)$ & $1.8-402$ & $223.05(199.26,249.68)$ & $15.41-8,188$ \\
\hline PCB 156 & $2.26 / 0.03$ & 82 & 1.68 & $10.14(9.47,10.87)$ & $1.6-62.1$ & $0.07(0.06,0.07)$ & $0.02-1.03$ \\
\hline PCB $169^{d}$ & $4.65 / 32.01$ & 84 & 11.19 & $31.66(29.66,33.79)$ & $3.3-172$ & $210.45(197.19,224.60)$ & $22.7-3,340.4$ \\
\hline
\end{tabular}

a $L O D$ Limit of detection for lipid adjusted congeners

${ }^{\mathrm{b}}$ LA Lipid adjusted

'NLA Non-lipid-adjusted

${ }^{\mathrm{d}}$ Measured in $\mathrm{fg} / \mathrm{g}$

When analyzing the population stratified above and below the median age, the directions of the associations for PCB 146, lead, and PCB 153 were similar and although the magnitudes of associations between age groups were different $(>10 \%)$ the strength of the associations were no longer significant (Table 4). The directions of associations were also similar when analyzing the population stratified by sex, although the magnitudes of associations between sexes were different $(>10 \%)$, specifically every neurotoxic compound had a greater magnitude of association for females in comparison with males, except for PCB 74. However only PCB 153 was significantly associated with cognitive functioning in females (Table 5). No significant interactions were observed for either sex or age (Table 6).

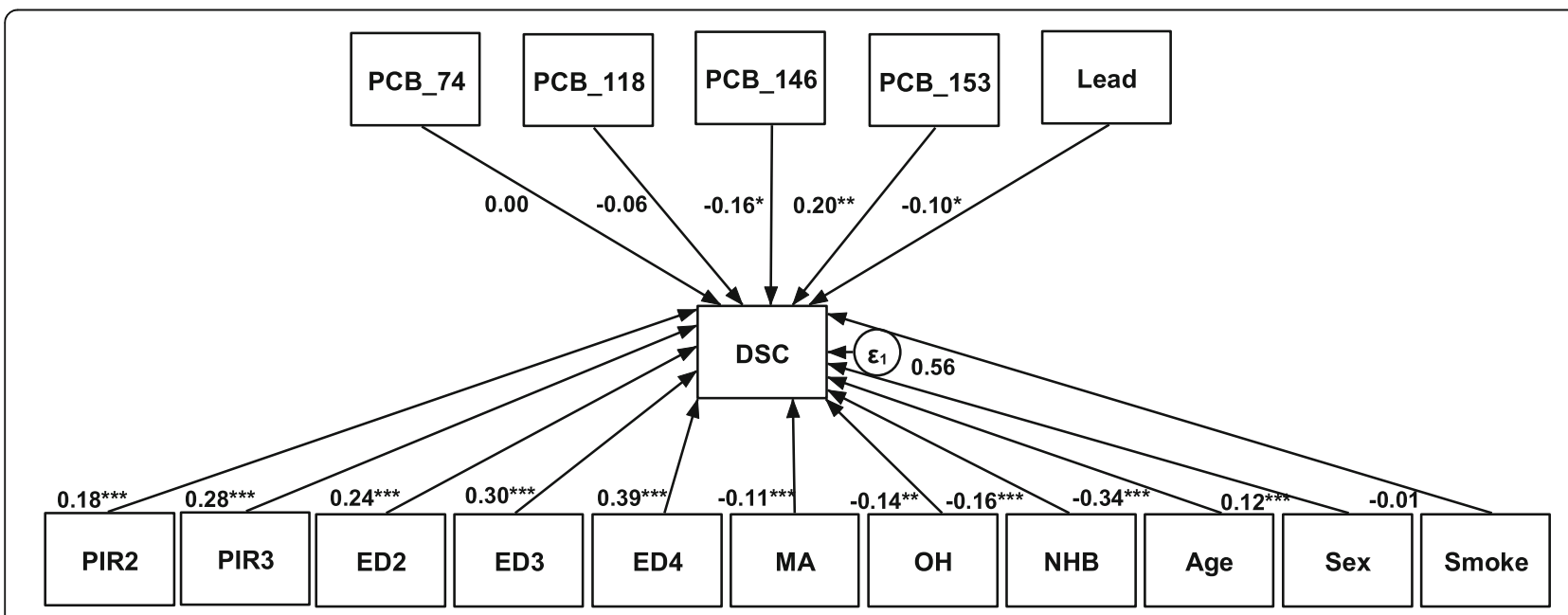

Fig. 2 Path analysis of relationship between DSC scores and environmental chemicals after adjusting for confounding factors. Males and females 60-84 years of age, NHANES 1999-2002. Ed1 is less than 9th grade, Ed2 is High School Grad/GED, Ed3 is Some College or AA degree, Ed4 is College Graduate or above, MA is Mexican American, $\mathrm{OH}$ is Other Hispanic and NHB is Non-Hispanic Black 
Table 3 Standardized path coefficients depicting the relationship between 5 chemicals, cognitive functioning and covariates for all participants NHANES 1999-2002 ( $N=498)$

\begin{tabular}{|c|c|c|c|c|}
\hline \multirow[t]{2}{*}{ Path } & \multicolumn{2}{|c|}{ Path coefficient $(95 \%$ Cl) } & \multicolumn{2}{|l|}{$p$-value } \\
\hline & Lipid-adjusted & Non-lipid-adjusted & Lipid-adjusted & Non-lipid-adjusted \\
\hline $\mathrm{PCB} 74 \rightarrow \mathrm{DSC}$ & $0.00(-0.13,0.13)$ & $-0.001(-0.14,0.13)$ & 0.99 & 0.99 \\
\hline PCB $118 \rightarrow$ DSC & $-0.06(-0.20,0.08)$ & $-0.06(-0.22,0.09)$ & 0.41 & 0.41 \\
\hline PCB $146 \rightarrow$ DSC & $-0.16(-0.29,-0.02)$ & $-0.17(-0.32,-0.02)$ & 0.02 & 0.03 \\
\hline PCB $153 \rightarrow$ DSC & $0.20(0.05,0.35)$ & $0.21(0.06,0.37)$ & 0.01 & 0.008 \\
\hline Lead $\rightarrow$ DSC & $-0.10(-0.20,-0.006)$ & $-0.10(-0.20,-0.007)$ & 0.04 & 0.04 \\
\hline${ }^{\mathrm{a} P I R 2} \rightarrow$ DSC & $0.18(0.06,0.30)$ & $0.18(0.06,0.30)$ & 0.003 & 0.003 \\
\hline${ }^{\mathrm{b}} \mathrm{PIR} 3 \rightarrow \mathrm{DSC}$ & $0.28(0.16,0.41)$ & $0.29(0.16,0.41)$ & $<0.001$ & $<0.001$ \\
\hline${ }^{\mathrm{C}} \mathrm{ED} 2 \rightarrow \mathrm{DSC}$ & $0.24(0.16,0.32)$ & $0.26(0.17,0.34)$ & $<0.001$ & $<0.001$ \\
\hline${ }^{d}$ ED3 $\rightarrow$ DSC & $0.30(0.22,0.38)$ & $0.24(0.16,0.32)$ & $<0.001$ & $<0.001$ \\
\hline${ }^{\mathrm{e}} \mathrm{ED} 4 \rightarrow \mathrm{DSC}$ & $0.39(0.27,0.52)$ & $0.30(0.22,0.38)$ & $<0.001$ & $<0.001$ \\
\hline${ }^{\mathrm{f}} \mathrm{MA} \rightarrow \mathrm{DSC}$ & $-0.11(-0.16,-0.06)$ & $-0.11(-0.16,-0.06)$ & $<0.001$ & $<0.001$ \\
\hline${ }^{9} \mathrm{OH} \rightarrow \mathrm{DSC}$ & $-0.14(-0.23,-0.06)$ & $-0.14(-0.23,-0.06)$ & 0.001 & 0.001 \\
\hline${ }^{\mathrm{h}} \mathrm{NHB} \rightarrow \mathrm{DSC}$ & $-0.16(-0.23,-0.10)$ & $-0.16(-0.22,-0.10)$ & $<0.001$ & $<0.001$ \\
\hline Age $\rightarrow$ DSC & $-0.31(-0.39,-0.24)$ & $-0.31(-0.39,-0.24)$ & $<0.001$ & $<0.001$ \\
\hline Sex $\rightarrow$ DSC & $0.12(0.03,0.20)$ & $0.12(0.03,0.10)$ & 0.007 & 0.007 \\
\hline Smoke $\rightarrow$ DSC & $-0.01(-0.12,0.09)$ & $-0.01(-0.12,0.10)$ & 0.85 & 0.85 \\
\hline
\end{tabular}

a PIR2 $2^{\text {nd }}$ Tertile Poverty Index Ratio

${ }^{b}$ PIR2 $3{ }^{\text {nd }}$ Tertile Poverty Index Ratio

${ }^{c} E d 2$ High School Grad/GED

${ }^{\mathrm{d}}$ Ed3 Some College or AA degree

${ }^{\mathrm{e}} \mathrm{Ed} 4$ College Graduate or above

${ }^{f} M A$ Mexican American

${ }^{9} \mathrm{OH}$ Other Hispanic

${ }^{\mathrm{h}} \mathrm{NHB}$ Non-Hispanic Black

Additionally, no substantial differences in the observed associations when PCBs were modeled using non-lipid adjusted PCBs (Tables 3, 4, and 5). The estimates generated when using multiple imputation for missing data were similar to estimates using complete case analysis. Because the models were saturated model fit is perfect with a Tucker Lewis Index (TLI) and Comparative Fit Index (CFI) of 1.00 and Root Mean Square Error of Approximation (RMSEA) and Standardized Root Mean Square Residual (RMSR) of 0.00 .

\section{Discussion}

In this cross sectional study of the U.S. population we observed that chemical exposures were associated with cognitive functioning in 60-84 year olds. After controlling for co-exposures, we identified PCB 146 as having a negative relationship with cognitive functioning. This is important because non-coplanar PCBs do not activate the arylhydrocarbon receptor (AhR) [11] and subsequently are less of a concern to regulatory bodies. We also observed that lead was weakly associated with lower cognitive scores in this older adult population which is not surprising given that lead is a known neurotoxic compound [42, 43]. This association has also been demonstrated in previous studies [17, 18, 44-46]. Lead can pass through the blood-brain barrier (BBB) by substituting for calcium ions and affect the brain through oxidative stress, altering neurotransmission, or inducing neural cells cell death [3-6]. There is also evidence from mouse studies that exposure to lead results in the accumulation of amyloid beta protein [47], which accumulates in the brain of Alzheimer's patients [48]. Interestingly, when we were building our model, lead was very strongly associated with lower cognitive scores until we controlled for smoking status which attenuated the strength of the association. This was not unexpected as tobacco smoke is a source of lead and has been shown to be a predictor of cognitive decline $[3,36,49]$.

Surprisingly, we did not find an association between cadmium and cognitive functioning despite cadmium being known neurotoxicant [50]. However, the relationship between cadmium and cognitive function in adults is not clear. For instance, several studies using various tests to assess cognitive functioning have found a negative relationship between cadmium exposure and cognitive functioning $[19,20]$. In contrast, a study using the MMSE in Malaysian $60-72$ year olds $(n=54)$ found a 
Table 4 Standardized path coefficients depicting the relationship between 5 chemicals, cognitive functioning and covariates for participants 60-69 and 70-84 years old, NHANES 1999-2002

\begin{tabular}{|c|c|c|c|c|}
\hline \multirow[t]{2}{*}{ Path } & \multicolumn{4}{|c|}{ Path coefficient (95\% Cl) p-value } \\
\hline & Lipid-adjusted & Non-lipid-adjusted & Lipid-adjusted & Non-lipid-adjusted \\
\hline \multicolumn{5}{|l|}{ Age 60-69 } \\
\hline $\mathrm{PCB} 74 \rightarrow \mathrm{DSC}$ & $0.01(-0.21,0.23)$ & $0.001(-0.23,0.23)$ & 0.92 & 0.99 \\
\hline PCB $118 \rightarrow$ DSC & $-0.10(-0.23,0.25)$ & $0.02(-0.23,0.27)$ & 0.93 & 0.89 \\
\hline PCB $146 \rightarrow$ DSC & $-0.20(-0.43,0.02)$ & $-0.21(-0.44,0.03)$ & 0.08 & 0.08 \\
\hline PCB $153 \rightarrow$ DSC & $0.28(0.03,0.53)$ & $0.28(0.02,0.54)$ & 0.03 & 0.04 \\
\hline Lead $\rightarrow$ DSC & $-0.13(-0.28,0.01)$ & $-0.13(-0.28,0.02)$ & 0.07 & 0.08 \\
\hline${ }^{\mathrm{a}} \mathrm{PIR} 2 \rightarrow \mathrm{DSC}$ & $0.16(0.03,0.30)$ & $0.16(0.03,0.29)$ & 0.02 & 0.02 \\
\hline${ }^{\mathrm{b} P I R 3} \rightarrow \mathrm{DSC}$ & $0.33(0.15,0.50)$ & $0.33(0.15,0.50)$ & $<0.001$ & $<0.001$ \\
\hline${ }^{\mathrm{c}} \mathrm{ED} 2 \rightarrow \mathrm{DSC}$ & $0.25(0.08,0.42)$ & $0.25(0.08,0.42)$ & 0.004 & 0.003 \\
\hline${ }^{\mathrm{d}} \mathrm{ED} 3 \rightarrow \mathrm{DSC}$ & $0.28(0.17,0.39)$ & $0.28(0.17,0.39)$ & $<0.001$ & $<0.001$ \\
\hline${ }^{\mathrm{e}} \mathrm{ED} 4 \rightarrow \mathrm{DSC}$ & $0.29(0.18,0.40)$ & $0.29(0.18,0.41)$ & $<0.001$ & $<0.001$ \\
\hline${ }^{\mathrm{f}} \mathrm{MA} \rightarrow \mathrm{DSC}$ & $-0.12(-0.20,-0.04)$ & $-0.12(-0.20,-0.05)$ & 0.003 & 0.002 \\
\hline${ }^{9} \mathrm{OH} \rightarrow \mathrm{DSC}$ & $-0.12(-0.26,0.02)$ & $-0.12(-0.26,-0.02)$ & 0.09 & 0.09 \\
\hline${ }^{\mathrm{h}} \mathrm{NHB} \rightarrow \mathrm{DSC}$ & $-0.18(-0.25,-0.10)$ & $-0.18(-0.25,-0.10)$ & $<0.001$ & $<0.001$ \\
\hline Age $\rightarrow$ DSC & $-0.13(-0.24,-0.01)$ & $-0.13(-0.24,-0.01)$ & 0.03 & 0.03 \\
\hline Sex $\rightarrow$ DSC & $0.14(0.05,0.24)$ & $0.14(0.04,0.23)$ & 0.004 & 0.005 \\
\hline Smoke $\rightarrow$ DSC & $0.04(-0.11,0.19)$ & $0.04(-0.11,0.19)$ & 0.58 & 0.60 \\
\hline \multicolumn{5}{|c|}{ Age $70-84(N=248)$} \\
\hline PCB $74 \rightarrow$ DSC & $-0.02(-0.21,0.18)$ & $-0.02(-0.22,0.18)$ & 0.88 & 0.88 \\
\hline PCB $118 \rightarrow$ DSC & $-0.13(-0.29,0.03)$ & $-0.13(-0.31,0.04)$ & 0.12 & 0.13 \\
\hline PCB $146 \rightarrow$ DSC & $-0.10(-0.41,0.21)$ & $-0.10(-0.44,0.23)$ & 0.53 & 0.55 \\
\hline PCB $153 \rightarrow$ DSC & $0.12(-0.14,0.38)$ & $0.13(-0.16,0.42)$ & 0.37 & 0.40 \\
\hline Lead $\rightarrow$ DSC & $-0.08(-0.20,0.04)$ & $-0.08(-0.20,0.04)$ & 0.19 & 0.17 \\
\hline $\mathrm{PIR} 2 \rightarrow \mathrm{DSC}$ & $0.19(0.03,0.35)$ & $0.19(0.02,0.35)$ & 0.02 & 0.03 \\
\hline $\mathrm{PIR} 3 \rightarrow \mathrm{DSC}$ & $0.26(0.11,0.42)$ & $0.26(0.10,0.41)$ & 0.001 & 0.001 \\
\hline $\mathrm{ED} 2 \rightarrow \mathrm{DSC}$ & $0.25(0.12,0.39)$ & $0.25(0.12,0.39)$ & $<0.001$ & $<0.001$ \\
\hline $\mathrm{ED} 3 \rightarrow \mathrm{DSC}$ & $0.18(0.04,0.32)$ & $0.18(0.03,0.32)$ & 0.01 & 0.02 \\
\hline $\mathrm{ED} 4 \rightarrow \mathrm{DSC}$ & $0.30(0.18,0.42)$ & $0.31(0.19,0.42)$ & $<0.001$ & $<0.001$ \\
\hline $\mathrm{MA} \rightarrow \mathrm{DSC}$ & $-0.11(-0.18,-0.05)$ & $-0.11(-0.17,-0.05)$ & $<0.001$ & $<0.001$ \\
\hline $\mathrm{OH} \rightarrow \mathrm{DSC}$ & $-0.20(-0.32,-0.09)$ & $-0.20(-0.31,-0.09)$ & $<0.001$ & $<0.001$ \\
\hline $\mathrm{NHB} \rightarrow \mathrm{DSC}$ & $-0.17(-0.27,-0.07)$ & $-0.18(-0.27,-0.08)$ & $<0.001$ & $<0.001$ \\
\hline Age $\rightarrow$ DSC & $-0.22(-0.30,-0.10)$ & $-0.20(-0.30,-0.10)$ & $<0.001$ & $<0.001$ \\
\hline Sex $\rightarrow$ DSC & $0.10(-0.04,0.24)$ & $0.11(-0.04,0.25)$ & 0.15 & 0.15 \\
\hline Smoke $\rightarrow$ DSC & $-0.06(-0.18,0.07)$ & $-0.06(-0.18,0.07)$ & 0.35 & 0.37 \\
\hline
\end{tabular}

${ }_{\text {a }}$ IR2 $2^{\text {nd }}$ Tertile Poverty Index Ratio

${ }^{b}$ PIR2 $3{ }^{\text {nd }}$ Tertile Poverty Index Ratio

${ }^{c}$ Ed2 High School Grad/GED

${ }^{\mathrm{d} E d 3}$ Some College or AA degree

${ }^{\mathrm{e}} \mathrm{Ed} 4$ College Graduate or above

${ }^{f} M A$ Mexican American

${ }^{9} \mathrm{OH}$ Other Hispanic

${ }^{\mathrm{h}} \mathrm{NHB}$ Non-Hispanic Black

non-significant positive relationship between cadmium exposure and cognitive functioning [51]. These discrepancies could be due to different effects of cadmium at different ages, controlling for different confounders, or study design. Further research is needed to determine the effect of cadmium on cognitive functioning in adults and the elderly. 
Table 5 Standardized path coefficients depicting the relationship between 5 chemicals, cognitive functioning and covariates for females and males age years old, NHANES 1999-2002

\begin{tabular}{|c|c|c|c|c|}
\hline \multirow[t]{2}{*}{ Path } & \multicolumn{4}{|c|}{ Path coefficient (95\% CI) p-value } \\
\hline & Lipid-adjusted & Non-lipid-adjusted & Lipid-adjusted & Non-lipid-adjusted \\
\hline \multicolumn{5}{|l|}{ Males $(N=231)$} \\
\hline $\mathrm{PCB} 74 \rightarrow \mathrm{DSC}$ & $0.03(-0.11,0.17)$ & $0.03(-0.13,0.18)$ & 0.66 & 0.74 \\
\hline PCB $118 \rightarrow$ DSC & $-0.01(-0.25,0.22)$ & $-0.002(-0.26,0.26)$ & 0.92 & 0.99 \\
\hline PCB $146 \rightarrow$ DSC & $-0.09(-0.31,0.13)$ & $-0.11(-0.36,0.14)$ & 0.42 & 0.38 \\
\hline PCB $153 \rightarrow$ DSC & $0.05(-0.22,0.32)$ & $0.05(-0.25,0.35)$ & 0.72 & 0.74 \\
\hline Lead $\rightarrow$ DSC & $-0.09(-0.24,0.06)$ & $-0.09(-0.23,0.06)$ & 0.23 & 0.23 \\
\hline${ }^{\mathrm{a}} \mathrm{PIR} 2 \rightarrow \mathrm{DSC}$ & $0.21(0.05,0.36)$ & $0.21(0.06,0.36)$ & 0.01 & 0.008 \\
\hline${ }^{\mathrm{b}} \mathrm{PIR3} \rightarrow \mathrm{DSC}$ & $0.39(0.21,0.58)$ & $0.39(0.21,0.58)$ & $<0.001$ & $<0.001$ \\
\hline${ }^{\mathrm{C}} \mathrm{ED} 2 \rightarrow \mathrm{DSC}$ & $0.20(0.10,0.30)$ & $0.20(0.11,0.30)$ & $<0.001$ & $<0.001$ \\
\hline${ }^{\mathrm{d}} \mathrm{ED} 3 \rightarrow \mathrm{DSC}$ & $0.25(0.15,0.36)$ & $0.25(0.14,0.36)$ & $<0.001$ & $<0.001$ \\
\hline${ }^{\mathrm{e}} \mathrm{ED} 4 \rightarrow \mathrm{DSC}$ & $0.35(0.20,0.50)$ & $0.35(0.20,0.50)$ & $<0.001$ & $<0.001$ \\
\hline${ }^{\mathrm{f}} \mathrm{MA} \rightarrow \mathrm{DSC}$ & $-0.10(-0.16,-0.03)$ & $-0.10(-0.16,-0.03)$ & 0.003 & 0.002 \\
\hline${ }^{9} \mathrm{OH} \rightarrow \mathrm{DSC}$ & $-0.17(-0.35,0.02)$ & $-0.17(-0.36,0.02)$ & 0.09 & 0.08 \\
\hline${ }^{h} \mathrm{NHB} \rightarrow \mathrm{DSC}$ & $-0.14(-0.23,-0.05)$ & $-0.14(-0.23,-0.05)$ & 0.003 & 0.002 \\
\hline Age $\rightarrow$ DSC & $-0.27(-0.36,-0.18)$ & $-0.27(-0.36,-0.18)$ & $<0.001$ & $<0.001$ \\
\hline Smoke $\rightarrow$ DSC & $0.05(-0.10,0.20)$ & $0.05(-0.10,0.21)$ & 0.51 & 0.51 \\
\hline \multicolumn{5}{|l|}{ Females $(N=267)$} \\
\hline $\mathrm{PCB} 74 \rightarrow \mathrm{DSC}$ & $0.005(-0.25,0.26)$ & $0.008(-0.24,0.26)$ & 0.97 & 0.95 \\
\hline PCB $118 \rightarrow$ DSC & $-0.12(-0.32,0.08)$ & $-0.12(-0.33,0.08)$ & 0.25 & 0.24 \\
\hline PCB $146 \rightarrow$ DSC & $-0.21(-0.45,0.03)$ & $-0.22(-0.46,0.03)$ & 0.08 & 0.08 \\
\hline PCB $153 \rightarrow$ DSC & $0.32(0.05,0.59)$ & $0.33(0.05,0.61)$ & 0.02 & 0.02 \\
\hline Lead $\rightarrow$ DSC & $-0.12(-0.26,0.01)$ & $-0.12(-0.26,0.01)$ & 0.08 & 0.05 \\
\hline $\mathrm{PIR} 2 \rightarrow \mathrm{DSC}$ & $0.15(0.000,0.31)$ & $0.16(0.002,0.31)$ & 0.05 & $<0.001$ \\
\hline $\mathrm{PIR} 3 \rightarrow \mathrm{DSC}$ & $0.19(0.03,0.34)$ & $0.19(0.03,0.34)$ & 0.02 & 0.0001 \\
\hline $\mathrm{ED} 2 \rightarrow \mathrm{DSC}$ & $0.28(0.15,0.41)$ & $0.28(0.15,0.41)$ & $<0.001$ & $<0.0001$ \\
\hline $\mathrm{ED} 3 \rightarrow \mathrm{DSC}$ & $0.23(0.09,0.36)$ & $0.23(0.09,0.36)$ & 0.001 & $<0.001$ \\
\hline $\mathrm{ED} 4 \rightarrow \mathrm{DSC}$ & $0.22(0.11,0.33)$ & $0.22(0.11,0.33)$ & $<0.001$ & 0.02 \\
\hline $\mathrm{MA} \rightarrow \mathrm{DSC}$ & $-0.13(-0.19,-0.06)$ & $-0.13(-0.19,-0.06)$ & $<0.001$ & $<0.001$ \\
\hline $\mathrm{OH} \rightarrow \mathrm{DSC}$ & $-0.15(-0.27,-0.03)$ & $-0.15(-0.27,-0.03)$ & 0.02 & 0.11 \\
\hline $\mathrm{NHB} \rightarrow \mathrm{DSC}$ & $-0.19(-0.27,-0.11)$ & $-0.19(-0.27,-0.11)$ & $<0.001$ & $<0.001$ \\
\hline Age $\rightarrow$ DSC & $-0.37(-0.48,-0.25)$ & $-0.37(-0.49,-0.25)$ & $<0.001$ & $<0.001$ \\
\hline Smoke $\rightarrow$ DSC & $-0.08(-0.22,0.06)$ & $-0.08(-0.22,0.06)$ & 0.28 & 0.28 \\
\hline
\end{tabular}

aPIR2 $2^{\text {nd }}$ Tertile Poverty Index Ratio

${ }^{\mathrm{b}}$ PIR3 $3^{\text {nd }}$ Tertile Poverty Index Ratio

${ }^{c}$ Ed2 High School Grad/GED

${ }^{\mathrm{d}}$ Ed3 Some College or AA degree

${ }^{e} E d 4$ College Graduate or above

${ }^{\mathrm{f}} \mathrm{MA}$ Mexican American

${ }^{9} \mathrm{OH}$ Other Hispanic

${ }^{\mathrm{h}} \mathrm{NHB}$ Non-Hispanic Black

Similar to previous studies using toxic equivalents, total PCBs or total dioxin-like PCBs, we found a significant negative relationship between non-dioxin like PCB 146 and cognitive functioning after controlling for co-exposure to four other PCB congeners [21, 28, 40,52, 53]. Unlike dioxin-like PCBs which activates the aryl hydrocarbon receptor (AhR), non-dioxin like PCBs do not activate the AhR [54]. Instead, epidemiology and animal toxicological studies suggest non-dioxin like PCBs act on the CNS by decreasing dopamine production [55-58].

Interestingly, we also observed that PCB 153 had a significant positive association with cognitive functioning. 
Table 6 Wald's Chi-Square Test Statistics and p-values testing equality of regression coefficients for chemical exposure and cognitive functioning between groups, NHANES 1999-2002

\begin{tabular}{llllc}
\hline Path & $\begin{array}{l}\text { Walds Test } \\
\text { Statistic }\end{array}$ & $p$-value & $\begin{array}{l}\text { Walds Test } \\
\text { Statistic }\end{array}$ & $p$-value \\
& Gender (male/female) & Age (above/below mean) \\
\hline PCB 74 $\rightarrow$ DSC & 0.01 & 0.91 & 0.08 & 0.78 \\
PCB 118 $\rightarrow$ DSC & 0.56 & 0.46 & 0.99 & 0.32 \\
PCB 146 $\rightarrow$ DSC & 0.62 & 0.43 & 0.44 & 0.51 \\
PCB 153 $\rightarrow$ DSC & 1.93 & 0.16 & 0.51 & 0.47 \\
Lead $\rightarrow$ DSC & 0.26 & 0.61 & 0.37 & 0.54
\end{tabular}

We are unaware of a biological mechanism that can explain the positive association between PCB 153 and cognitive functioning. Further path analysis examining PCB 153 as the sole exposure variable resulted in a non-significant positive relationship between PCB 153 and cognitive functioning, suggesting the significance of individual exposures may differ than when the compound is examined in a mixture. Additionally, PCB 153 has a less potent Neurotoxic Equivalent (NEQ) than PCB 146. NEQs are determined using REPs that are derived using in vitro experiments with neurotoxic outcomes. NEQs were developed to account for the neurotoxicity of PCB congeners, such as ortho-substituted non-coplanar PCBs, which are not included in the TEF scheme [59]. Similarly to modeling sum PCBs, modeling PCB toxic equivalents could overestimate or underestimate risk of altered cognitive functioning by not addressing PCBs' neurotoxic mode of action.

There are several strength to this study. Specifically, we were able to simultaneous control for the multiple comparisons of several chemical exposures representing different classes of persistent environmental pollutants and other confounders. However, there are several limitations that are worth mentioning. NHANES is a crosssectional study which prevents us from understanding the temporality between the exposures and the outcomes. Although, the environmental exposure used in this analysis have relatively long half-lives on the order of months to years [60-62] and therefore represent long-term exposure. Additionally, the DSC scores have proven to be a strong predictor of cognitive functioning even when assessed at only one time point [63]. Also we were unable to account for several important covariates. For instance, data was not available on neurotoxic and neuroprotective factors such as methylmercury [64] and omega-3 fatty acids [65], respectively. NHANES only measures a subset of chemicals in each participant which limits the number of compounds that can be included in this analysis. Therefore, we were unable to model additional chemicals which may play a synergistic or antagonistic role in the association between environmental exposures and cognitive functioning. Finally, findings may not be representative of the general population because the analyzed population had a different racial composition compared to the population that was excluded due to missing data.

\section{Conclusion}

In this sample of older US adults, we observed a dosedepended effect between lower cognitive functioning and non-dioxin like PCBs and metals, specifically PCB 146 and lead. Additional animal toxicity and epidemiology are needed to confirm the role of non-dioxin like PCBs and declines in cognitive function. Continued development and incorporation of the recently proposed NEQs will allow the impact of non-dioxin like PCBs on neurotoxicity to be properly addressed. Since neurodegenerative disease have a high economic and social burden and many environmental exposures are preventable the potential impact of exposure to neurotoxic compounds in late life is great and warrants further investigation to guide prevention and intervention efforts.

\section{Abbreviations \\ AhR: Arylhydrocarbon receptor; CDC: Center for disease control; CERAD: Consortium to establish a registry for alzheimer's disease; CNS: Central nervous system; CSID: Community screening instrument for dementia; DSC: Digit symbol coding; ICP-MS: Inductively coupled plasma mass spectrometry; IU: Indiana University; LOD: Limit of detection; MA: Mexican American; MCl: Mild cognitive impairment; MMSE: Mini mental state exam; NCHS: National Center for Health Statistics; NEQ: Neurotoxic equivalent; NHANES: National Health and Nutrition Examination Survey; NHB: Non-hispanic black; NHW: Non-hispanic white; OH: Other hispanic; PCBs: Polychlorinated biphenyls; PIR: Poverty index ratio; REP: Relative effect potencies; SDST: Symbol digit substitution test; TEFs: Toxic equivalency factors; VA: Veterans affair; WASI-III: Weschler adult intelligence scale, $3^{\text {rd }}$ edition; WHO: World Health Organization}

\section{Acknowledgements}

The authors would like to thank Dr. Robert Tanguay for his input on study design.

\section{Funding}

This work was supported by grants from the US National Institute of Environmental Health Sciences T32 ES007060 and P30 ES000210.

\section{Availability of data and materials}

The datasets generated during and/or analyzed during the current study are available in the NHANES repository, http://www.cdc.gov/nchs/nhanes/ search/nhanes99_00.aspx and http://www.cdc.gov/nchs/nhanes/search/ nhanes01_02.aspx.

\section{Authors' contributions}

JP conceived the study design. AH, ES, and MK assisted with development of statistical analysis plan. The analysis was conducted by JP. The paper was jointly written by JP and MK. All authors read and approved the final manuscript.

\section{Competing interests}

The authors declare that they have no competing interests

Consent for publication

Not applicable 


\section{Ethics approval and consent to participate}

All protocols were approved by the National Center for Health Statistics (NCHS) Research Ethics Review Board (ERB) and documented consent was obtained from all participants.

\section{Received: 12 October 2016 Accepted: 28 February 2017 Published online: 07 March 2017}

\section{References}

1. Méndez-Armenta M, Ríos C. Cadmium neurotoxicity. Environ Toxicol Pharmacol. 2007;23:350-8.

2. Seegal RF. Epidemiological and laboratory evidence of PCB-Induced neurotoxicity. Crit Rev Toxicol. 1996;26:709-37.

3. Sanders T, Liu Y, Buchner V, Tchounwou PB. Neurotoxic effects and biomarkers of lead exposure: a review. Rev Environ Health. 2009;24:15-46.

4. Jomova K, Valko M. Advances in metal-induced oxidative stress and human disease. Toxicology. 2011;283:65-87.

5. Nemsadze K, Sanikidze T, Ratiani L, Gabunia L, Sharashenidze T. Mechanisms of lead-induced poisoning. Georgian Med News. 2008;172-173:92-6.

6. Engstrom A, Wang H, Xia ZG. Lead decreases cell survival, proliferation, and neuronal differentiation of primary cultured adult neural precursor cells through activation of the JNK and p38 MAP kinases. Toxicol in Vitro. 2015; 29:1146-55

7. United States Department of Health and Human Services (USDHH). 2011. Promoting Brain Health. Available: http://www.cdc.gov/aging/pdf/cognitive_ impairment/cogimp genaud final.pdf. Accessed 25 Sept 2014.

8. Knickman JR, Snell EK. The 2030 problem: caring for aging baby boomers. Health Serv Res. 2002;37:849-84.

9. Mahoney R, Regan C, Katona C, Livingston G. Anxiety and depression in family caregivers of people with Alzheimer disease: the LASER-AD study. Am J Geriatr Psychiatry. 2005;13:795-801.

10. Plassman BL, Langa KM, Fisher GG, Heeringa SG, Weir DR, Ofstedal MB, Burke JR, Hurd MD, Potter GG, Rodgers WL. Prevalence of dementia in the United States: the aging, demographics, and memory study. Neuroepidemiology. 2007;29:125-32.

11. Winneke G, Bucholski A, Heinzow B, Krämer U, Schmidt E, Walkowiak J, Wiener J-A, Steingrüber H-J. Developmental neurotoxicity of polychlorinated biphenyls (PCBS): cognitive and psychomotor functions in 7-month old children. Toxicol Lett. 1998;102:423-8.

12. Bushnell PJ, Moser VC, MacPhail RC, Oshiro WM, Derr-Yellin EC, Phillips PM, Kodavanti PRS. Neurobehavioral assessments of rats perinatally exposed to a commercial mixture of polychlorinated biphenyls. Toxicol Sci. 2002;68:109-20.

13. Chopra V, Harley K, Lahiff M, Eskenazi B. Association between phthalates and attention deficit disorder and learning disability in U.S. children, 6-15 years. Environ Res. 2014;128:64-9.

14. Grandjean P, Landrigan PJ. Developmental neurotoxicity of industrial chemicals. Lancet. 2006;368:2167-78.

15. Andersen HR, Nielsen JB, Grandjean P. Toxicologic evidence of developmental neurotoxicity of environmental chemicals. Toxicology. 2000;144:121-7.

16. Geller AM, Zenick $H$. Aging and the environment: a research framework. Environ Health Perspect. 2005;113:1257.

17. Payton M, Riggs KM, Spiro A, Weiss ST, Hu H. Relations of bone and blood lead to cognitive function: the VA normative aging study. Neurotoxicol Teratol. 1998:20:19-27.

18. Wright RO, Tsaih SW, Schwartz J, Avron Spiro III, McDonald K, Weiss ST, Hu H. Lead exposure biomarkers and mini-mental status exam scores in older men. Epidemiology. 2003;14:713-8.

19. Ciesielski T, Bellinger DC, Schwartz J, Hauser R, Wright RO. Associations between cadmium exposure and neurocognitive test scores in a crosssectional study of US adults. Environ Health. 2013;12:13.

20. Gao S, Jin Y, Unverzagt FW, Ma F, Hall KS, Murrell JR, Cheng Y, Shen J, Ying B, Ji R. Trace element levels and cognitive function in rural elderly Chinese. J Gerontol A Biol Sci Med Sci. 2008;63:635-41.

21. Fitzgerald EF, Belanger EE, Gomez Ml, Cayo M, McCaffrey RJ, Seegal RF, Jansing RL, Hwang S-A, Hicks HE. Polychlorinated biphenyl exposure and neuropsychological status among older residents of upper Hudson River communities. Environ Health Perspect. 2008;116:209.

22. Van den Berg M, Birnbaum LS, Denison M, De Vito M, Farland W, Feeley M, Fiedler H, Hakansson H, Hanberg A, Haws L. The 2005 World Health Organization reevaluation of human and mammalian toxic equivalency factors for dioxins and dioxin-like compounds. Toxicol Sci. 2006;93:223-41.
23. Van den Berg M, Birnbaum $L$, Bosveld $A$, Brunström B, Cook P, Feeley $M$, Giesy JP, Hanberg A, Hasegawa R, Kennedy SW. Toxic equivalency factors (TEFs) for PCBs, PCDDs, PCDFs for humans and wildlife. Environ Health Perspect. 1998;106:775.

24. Safe SH. Polychlorinated biphenyls (PCBs): environmental impact, biochemical and toxic responses, and implications for risk assessment. Crit Rev Toxicol. 1994;24:87-149.

25. Acock A. Discovering structural equation modeling using Stata. College Station: Stata Press Books; 2013.

26. Wright S. Correlation and causation. J Agric Res. 1921;20:557-85.

27. National Center for Health Statistics (NCHS). 2014. NHANES 2003-2004 Dietary Data. Available: http://wwwn.cdc.gov/nchs/nhanes/search/datapage. aspx?Component=Dietary\&CycleBeginYear=2003 [Accessed 2 Sept 2014].

28. Bouchard MF, Oulhote Y, Sagiv SK, Saint-Amour D, Weuve J. Polychlorinated biphenyl exposures and cognition in older U.S. Adults: NHANES (19992002). Environ Health Perspect. 2014;122:73-8.

29. Rudolf SMCRLL, Winkensa WHMPI, Verheya JJFRJ. Cognitive functioning after stroke: a one-year follow-up study. Dement Geriatr Cogn Disord. 2004;18:138-44.

30. Center for Disease Control and Prevention (CDC). Laboratory Procedure Manual: PCBs and Persistent Pesticides. Available: http://www.cdc.gov/nchs/data/nhanes/ nhanes_99_00/lab28poc_met_pcb_pesticides.pdf [Accessed 2 Aug 2016].

31. Center for Disease Control and Prevention (CDC). 2004. Laboratory Procedure Manual Analyte: Lead Cadmium Mercury. Available: http://www. cdc.gov/nchs/data/nhanes/nhanes_05_06/pbcd_d_met_lead_cadmium.pdf [Accessed 14 July 2016].

32. National Center for Health Statistics (NCHS). 2005. 2001-2002 Data Documentation, Codebook, and Frequencies: Cognitive Functioning Available: http://wwwn.cdc.gov/nchs/nhanes/2001-2002/CFQ_B.htm [Accessed 4 Dec 2014].

33. Paschal DC, Burt V, Caudill SP, Gunter EW, Pirkle JL, Sampson EJ, Miller DT, Jackson RJ. Exposure of the US population aged 6 years and older to cadmium: 1988-1994. Arch Environ Contam Toxicol. 2000;38:377-83.

34. Silva MJ, Barr DB, Reidy JA, Malek NA, Hodge CC, Caudill SP, Brock JW, Needham LL, Calafat AM. Urinary levels of seven phthalate metabolites in the US population from the National Health and Nutrition Examination Survey (NHANES) 1999-2000. Environ Health Perspect. 2004;112:331-8.

35. Tyrrell J, Melzer D, Henley W, Galloway TS, Osborne NJ. Associations between socioeconomic status and environmental toxicant concentrations in adults in the USA: NHANES 2001-2010. Environ Int. 2013;59:328-35.

36. Kalcher K, Kern W, Pietsch R. Cadmium and lead in the smoke of a filter cigarette. Sci Total Environ. 1993;128:21-35.

37. Chen R, Wilson K, Chen Y, Zhang D, Qin X, He M, Hu Z, Ma Y, Copeland JR. Association between environmental tobacco smoke exposure and dementia syndromes. Occup Environ Med. 2013;70:63-9.

38. Benowitz NL. Cotinine as a biomarker of environmental tobacco smoke exposure. Epidemiol Rev. 1996;18:188-204.

39. Johnson C, Paulose-Ram R, Ogden C. National Health and Nutrition Examination Survey: analytic guidelines, 1999-2010. 2013

40. Haase RF, McCaffrey RJ, Santiago-Rivera AL, Morse GS, Tarbell A. Evidence of an age-related threshold effect of polychlorinated biphenyls (PCBs) on neuropsychological functioning in a native American population. Environ Res. 2009;109:73-85.

41. Phillips DL, Pirkle JL, Burse WW, Bernert Jr JT, Henderson LO, Needham LL. Chlorinated hydrocarbon levels in human serum: effects of fasting and feeding. Arch Environ Contam Toxicol. 1989;18:495-500.

42. Bressler JP, Goldstein GW. Mechanisms of lead neurotoxicity. Biochem Pharmacol. 1991:41:479-84

43. Garza A, Vega R, Soto E. Cellular mechanisms of lead neurotoxicity. Med Sci Monit. 2006;12:RA57-65

44. Weisskopf MG, Proctor SP, Wright RO, Schwartz J, Spiro lii A, Sparrow D, Nie $\mathrm{H}, \mathrm{Hu} \mathrm{H}$. Cumulative lead exposure and cognitive performance among elderly men. Epidemiology. 2007;18:59-66.

45. Chia SE, Chia HP, Ong CN, Jeyaratnam J. Cumulative blood lead levels and neurobehavioral test performance. Neurotoxicology. 1996;18:793-803.

46. Schwartz BS, Lee B-K, Lee G-S, Stewart WF, Lee S-S, Hwang K-Y, Ahn K-D, Kim Y-B, Bolla Kl, Simon D. Associations of blood lead, dimercaptosuccinic acid-chelatable lead, and tibia lead with neurobehavioral test scores in South Korean lead workers. Am J Epidemiol. 2001:153:453-64.

47. Gu H, Robison G, Hong L, Barrea R, Wei X, Farlow MR, Pushkar YN, Du Y, Zheng $W$. Increased $\beta$-amyloid deposition in Tg-SWDI transgenic mouse brain following in vivo lead exposure. Toxicol Lett. 2012;213:211-9. 
48. Ogomori K, Kitamoto T, Tateishi J, Sato Y, Suetsugu M, Abe M. $\beta$-protein amyloid is widely distributed in the central nervous system of patients with Alzheimer's disease. Am J Pathol. 1989;134:243-51.

49. Nie H, Hu H, Weuve JL, Korrick SA, Weisskopf MG, Ryan LM, Schwartz JD, Grodstein F. Cumulative exposure to lead in relation to cognitive function in older women. 2008.

50. Wang B, Du Y. Cadmium and its neurotoxic effects. Oxidative Med Cell Longev. 2013;2013:898034.

51. Ghazali AR, Kamarulzaman F, Normah CD, Ahmad M, Ghazali SE, Ibrahim N, Said Z, Shahar S, Angkat N, Razali R. Levels of metallic elements and their potential relationships to cognitive function among elderly from federal land development authority (FELDA) settlement in Selangor Malaysia. Biol Trace Elem Res. 2013;153:16-21.

52. Lin K, Guo N, Tsai P, Yang C, Guo YL. Neurocognitive changes among elderly exposed to PCBs/PCDFs in Taiwan. Environ Health Perspect. 2008;1 16:184.

53. Schantz SL, Gasior DM, Polverejan E, McCaffrey RJ, Sweeney AM, Humphrey $\mathrm{HE}$, Gardiner JC. Impairments of memory and learning in older adults exposed to polychlorinated biphenyls via consumption of Great Lakes fish. Environ Health Perspect. 2001;109:605-11.

54. Safe S, Bandiera S, Sawyer T, Robertson L, Safe L, Parkinson A, Thomas PE, Ryan DE, Reik LM, Levin W. PCBs: structure-function relationships and mechanism of action. Environ Health Perspect. 1985;60:47.

55. Shain W, Bush B, Seegal R. Neurotoxicity of polychlorinated biphenyls: structure-activity relationship of individual congeners. Toxicol Appl Pharmacol. 1991;111:33-42.

56. Seegal RF, Bush B, Shain W. Lightly chlorinated ortho-substituted PCB congeners decrease dopamine in nonhuman primate brain and in tissue culture. Toxicol Appl Pharmacol. 1990;106:136-44.

57. Seegal RF, Marek KL, Seibyl JP, Jennings DL, Molho ES, Higgins DS, Factor $S A$, Fitzgerald EF, Hills EA, Korrick SA. Occupational exposure to PCBs reduces striatal dopamine transporter densities only in women: A $\beta$-CIT imaging study. Neurobiol Dis. 2010;38:219-25.

58. Mariussen E, Andersen JM, Fonnum F. The effect of polychlorinated biphenyls on the uptake of dopamine and other neurotransmitters into rat brain synaptic vesicles. Toxicol Appl Pharmacol. 1999:161:274-82.

59. Simon T, Britt JK, James RC. Development of a neurotoxic equivalence scheme of relative potency for assessing the risk of PCB mixtures. Regul Toxicol Pharmacol. 2007;48:148-70.

60. Leggett RW. An age-specific kinetic model of lead metabolism in humans. Environ Health Perspect. 1993;101:598.

61. Agency for Toxic Substances and Disease Registry (ATSDR). 2012. Public Health Statement: Cadmium. Available: http://www.atsdr.cdc.gov/ ToxProfiles/tp5-c1-b.pdf [Accessed 5 Nov 2015].

62. Wolff MS, Fischbein A, Thornton J, Rice C, Lilis R, Selikoff I. Body burden of polychlorinated biphenyls among persons employed in capacitor manufacturing. Int Arch Occup Environ Health. 1982:49:199-208.

63. Wechsler D. Wechsler adult intelligence scale-fourth edition (WAIS-IV). San Antonio: NCS Pearson; 2008.

64. Harris HH, Pickering IJ, George GN. The chemical form of mercury in fish. Science. 2003:301:1203.

65. van Gelder BM, Tijhuis M, Kalmijn S, Kromhout D. Fish consumption, $n-3$ fatty acids, and subsequent 5-y cognitive decline in elderly men: the Zutphen Elderly study. Am J Clin Nutr. 2007;85:1142-7.

\section{Submit your next manuscript to BioMed Central and we will help you at every step:}

- We accept pre-submission inquiries

- Our selector tool helps you to find the most relevant journal

- We provide round the clock customer support

- Convenient online submission

- Thorough peer review

- Inclusion in PubMed and all major indexing services

- Maximum visibility for your research

Submit your manuscript at www.biomedcentral.com/submit 